\title{
Endosonographic features predictive of benign and malignant gastrointestinal stromal cell tumours
}

\author{
L Palazzo, B Landi, C Cellier, E Cuillerier, G Roseau, J-P Barbier
}

\begin{abstract}
BackgroundlAim-Some endoscopic ultrasonographic (EUS) features have been reported to be suggestive of malignancy in gastrointestinal stromal cell tumours (SCTs). The aim of this study was to assess the predictive value of these features for malignancy.

Methods-A total of 56 histologically proven cases of SCT studied by EUS between 1989 and 1996 were reviewed. There were 42 gastric tumours, 12 oesophageal tumours, and two rectal tumours. The tumours were divided into two groups: (a) benign SCT, comprising benign leiomyoma $(n=34) ;(b)$ malignant or borderline SCT $(n=22)$, comprising leiomyosarcoma $(n=9)$, leiomyoblastoma ( $n$ = 9), and leiomyoma of uncertain malignant potential $(n=4)$. The main EUS features recorded were tumour size, ulceration, echo pattern, cystic spaces, extraluminal margins, and lymph nodes with a malignant pattern. The two groups were compared by univariate and multivariate analysis.
\end{abstract}

Results-Irregular extraluminal margins, cystic spaces, and lymph nodes with a malignant pattern were most predictive of malignant or borderline SCT. Pairwise combinations of the three features had a specificity and positive predictive value of $100 \%$ for malignant or borderline SCT, but a sensitivity of only $23 \%$. The presence of at least one of these three criteria had $91 \%$ sensitivity, $88 \%$ specificity, and $83 \%$ predictive positive value. In multivariate analysis, cystic spaces and irregular margins were the only two features independently predictive of malignant potential.

Department of Gastroenterology, Laennec Hospital and Clinique Turin, Paris, France

L Palazzo

B Landi

C Cellier

E Cuillerier

G Roseau

J-P Barbier

Correspondence to:

Dr B Landi, Department of

Gastroenterology, Laennec

Hospital, 42 rue de Sèvres,

75007 Paris, France.

Accepted for publication 3 August 1999
The features most predictive of benign SCTs were regular margins, tumour size $\leqslant 30 \mathrm{~mm}$, and a homogeneous echo pattern. When the three features were combined, histology confirmed a benign SCT in all cases.

Conclusions-The combined presence of two out of three EUS features (irregular extraluminal margins, cystic spaces, and lymph nodes with a malignant pattern) had a positive predictive value of $100 \%$ for malignant or borderline gastrointestinal SCT. Tumours less than $30 \mathrm{~mm}$ in diameter with regular margins and a homogeneous echo pattern are usually benign. (Gut 2000;46:88-92)
Keywords: gastrointestinal; stromal cell tumours; endoscopic ultrasonography; leiomyoma; leiomyoblastoma; leiomyosarcoma

Stromal cell tumours (SCTs) are the most common tumours of the oesophagogastric submucosa. ${ }^{12}$ Most gastrointestinal SCTs are asymptomatic and benign, but around $15 \%$ are malignant. ${ }^{1-3}$

Endoscopic ultrasonography (EUS) is the most appropriate technique for studying tumours of the oesophagogastric submucosa. EUS diagnosis of SCT is based on a hypoechoic mass contiguous with the fourth hypoechoic layer (muscularis propria) or the second hypoechoic layer (muscularis mucosae) of the normal wall. ${ }^{45}$ Several authors have reported that certain EUS features of SCTs may be predictive of malignancy. ${ }^{36}$ However, the studies were small and/or multicentre series, and the ability of EUS to distinguish between benign and malignant SCTs remained to be confirmed.

The aim of this study was therefore to identify EUS features distinguishing benign from malignant gastrointestinal SCTs in a large single centre population.

\section{Patients and methods}

From March 1989 to December 1997, the files of all patients with histologically proven leiomyoma, leiomyoblastoma, or leiomyosarcoma who underwent EUS examination in our endoscopic unit were reviewed. There were 56 patients (22 men and 34 women), with a mean age of 61 years (range 28-92). There were 42 gastric tumours, 12 oesophageal tumours, and two rectal tumours. The histological specimens were obtained by surgical resection in 50 cases $(89 \%)$, by endoscopic resection in three cases $(5.5 \%)$, and by endoscopic or surgical biopsy in three cases $(5.5 \%)$. Thirty eight leiomyomas, nine leiomyoblastomas, and nine leiomyosarcomas were diagnosed. Of the 38 leiomyomas, 34 were considered benign. Four were considered to be of indeterminate malignancy (borderline tumours), because of mitotic activity or pronounced pleomorphism and dense cellularity. ${ }^{12}$

A total of 54 of 56 patients had undergone an upper gastrointestinal endoscopy and two a rectosigmoidoscopy with biopsies of the submucosal tumour some days or weeks before EUS. The EUS examinations were performed

Abbreviations used in this paper: EUS, endoscopic ultrasonography; SCT, stromal cell tumour. 
Table 1 Comparison of endoscopic ultrasonographic (EUS) features in benign stromal cell tumours (SCTs) and SCTs with malignant potential (univariate analysis)

\begin{tabular}{|c|c|c|c|c|c|}
\hline Features & $\begin{array}{l}\text { Borderline SCTs } \\
(n=13)\end{array}$ & $\begin{array}{l}\text { Leiomyosarcomas } \\
(n=9)\end{array}$ & $\begin{array}{l}\text { SCTs with malignant } \\
\text { potential (borderline tumours } \\
+ \text { leiomyosarcoma) } \\
(n=22)\end{array}$ & $\begin{array}{l}\text { Benign leiomyomas } \\
(n=34)\end{array}$ & $\begin{array}{l}\text { SCTs with } \\
\text { malignant potential } \\
\text { versus benign } \\
\text { leiomyomas } \\
\text { ( } p \text { value) }\end{array}$ \\
\hline Maximal diameter $>30 \mathrm{~mm}$ & $10(77)$ & $7(78)$ & $17(77)$ & $14(41)$ & 0.003 \\
\hline Ulceration & $5(38)$ & $7(78)$ & $12(54)$ & $10(29)$ & 0.06 \\
\hline Lymph nodes & $1(8)$ & $4(44)$ & $5(23)$ & $1(3)$ & 0.02 \\
\hline Heterogeneity with cystic spaces & $6(46)$ & $3(33)$ & $9(41)$ & $2(6)$ & 0.001 \\
\hline Heterogeneity without cystic spaces & $3(23)$ & $3(33)$ & $6(27)$ & $11(32)$ & 0.9 \\
\hline Exophytic development & $6(46)$ & $2(22)$ & $8(36)$ & $4(12)$ & 0.03 \\
\hline Extraluminal irregular margins & $6(46)$ & $5(56)$ & $11(50)$ & $2(6)$ & 0.0002 \\
\hline
\end{tabular}

Values in parentheses are percentages.

after upper gastrointestinal endoscopy (Olympus XV-100 or GIF-100) without biopsies (except for four patients) in a single institution using instruments with radial transducers of 7.5 and $12 \mathrm{MHZ}$ (Olympus GF-UM 3 or GF-UM 20) (Scop Medecine Olympus, Rungis, France). All examinations were performed under intravenous sedation (midazolam with or without propofol).

The endoscopic and EUS charts, EUS photographs $(n=23)$, or videotapes when available $(n=33)$ were reviewed by one of us (LP) blinded to the final diagnosis. The following EUS features were recorded for all tumours: (a) maximal diameter; (b) presence of deep ulceration on endoscopy and/or EUS; (c) echogenicity (hypoechoic or hyperechoic); (d) heterogeneity (homogeneous $v$ heterogeneous); (e) presence of cystic spaces (anechoic cavities with posterior enhancement); $(f)$ regularity of the extraluminal border (regular $v$ irregular); $(g)$ exophytic development (bulk of tumour development outside the intestinal wall); (h) lymph nodes with malignant patterns (spherical, hypoechoic, sharply demarcated, largest diameter $>1 \mathrm{~cm}$ ).

To identify EUS features predictive of malignancy, the tumours were divided into two groups: A ( $\mathrm{n}=34)$, benign SCTs-that is, benign leiomyomas; $B(n=22)$, malignant SCTs - that is, leiomyosarcomas $(n=9)$ - and SCTs of uncertain malignant potential ( $\mathrm{n}=$ 13). SCTs of uncertain malignant potential corresponded to the nine leiomyoblastomas and four leiomyomas for which a benign nature could not be determined on the pathological specimens.

STATISTICAL ANALYSIS

The EUS features were compared between group $\mathrm{A}$ and group $\mathrm{B}$. Univariate analyses using the $\chi^{2}$ test were performed to identify EUS features associated with potential malignancy with the Statview IV statistical package. Maximal diameter of the tumour was considered to be a qualitative datum (more or less than $30 \mathrm{~mm}$ ). Multivariate logistic regression was performed to identify independent predictors of potential malignancy (JMP statistical package). $p$ values of 0.05 or less were considered statistically significant. Calculation of sensitivity, specificity, and negative and positive predictive values of each EUS feature and combination of these features for predicting potential malignancy were carried out manually. The same calculation of sensitivity,

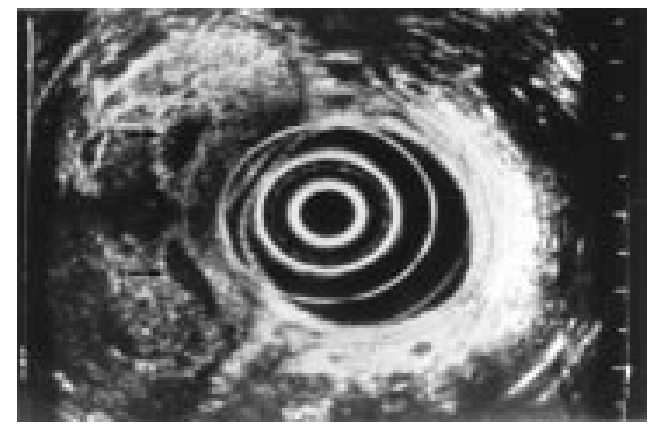

Figure 1 Gastric leiomyosarcoma (diameter $5 \mathrm{~cm}$ ) with several cystic spaces (arrows).

specificity, and negative and positive predictive values was performed for predicting the benignity of the tumour.

\section{Results}

ENDOSCOPIC AND EUS FEATURES

Table 1 shows the main EUS features of the tumours.

\section{Leiomyosarcomas $(n=9)$}

Leiomyosarcomas were located in the oesophagus in one patient $(11 \%$; accounting for $8 \%$ of oesophageal SCTs), in the stomach in six $(67 \% ; 14 \%$ of gastric SCT), and in the rectum in two $(22 \%$; all cases of rectal SCT). The mean maximal tumour diameter was 60 (range 26-100) $\mathrm{mm}$. The tumour was ulcerated in seven cases $(77 \%)$.

The tumours arose from the fourth echo poor layer in eight cases; EUS showed that one oesophageal tumour disrupted all the wall layers. Cystic spaces (fig 1) and irregular tumour margins (figs 2 and 3) were observed in three and five cases respectively (56\%). The tumour echostructure and margins were homogeneous and regular in three cases each (33\%), but only one patient had a homogeneous tumour with regular margins. In this latter case the tumour measured $40 \mathrm{~mm}$ in diameter. Involved satellite lymph nodes were present in four patients $(44 \%)$, and were large nodes located in the coeliac or hepatic area in two cases; they were diagnosed by transabdominal ultrasonography before the gastric tumour was found. At least one of the following features was present in eight of nine leiomyosarcomas $(89 \%)$ : irregular margins, cystic spaces, lymph nodes with malignant patterns.

Benign leiomyomas $(n=34)$

Benign leiomyomas were located in the oesophagus in 11 patients $(32 \% ; 92 \%$ of 


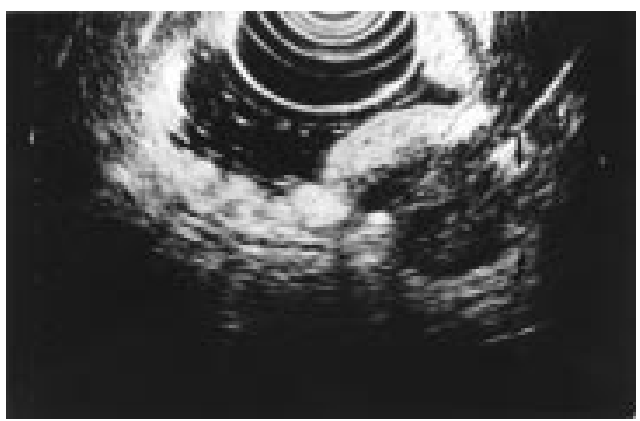

Figure 2 Small gastric leiomyosarcoma (diameter $2 \mathrm{~cm}$ ) with irregular margins (arrows)

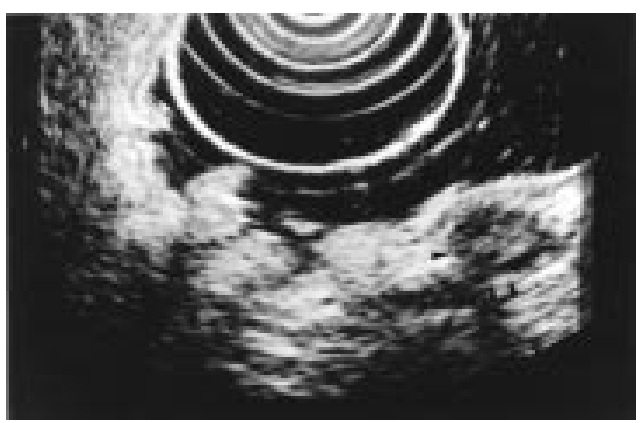

Figure 3 The same lesion as in fig 2. Note the lateral involvement of the submucosa (arrowheads).

oesophageal SCTs) and in the stomach in 23 $(68 \% ; 55 \%$ of gastric SCTs). The mean maximal diameter of the tumour was 34 (range $8-75) \mathrm{mm}$. Ten tumours (29\%) were ulcerated.

These tumours arose from or within the fourth echo poor layer in most cases ( $n=29$; $87 \%)$. Only five tumours (13\%) arose from the second echo poor layer, and all five were leiomyomas of the muscularis mucosae. The echostructure was homogeneous in $58 \%$ of cases, and $60 \%$ of the 20 tumours measured less than $30 \mathrm{~mm}$ in diameter. One or several cystic spaces were observed in two benign leiomyomas (6\%) measuring 40 and $60 \mathrm{~mm}$. These spaces corresponded to cystic degeneration in one case, as shown by histological examination. In the other case, major intratumoral oedema was observed on the pathological specimen, without necrosis or cystic degeneration. Hyperechoic spots, corresponding histologically to calcifications, were observed in three cases $(8 \%)$. Tumour margins were irregular in two leiomyomas (6\%) measuring 25 and $35 \mathrm{~mm}$. Lymph nodes with a malignant pattern were present in one case of gastric leiomyoma located at the greater curve. They were not adjacent to the tumour but had a metastatic pattern. In fact, they were metastatic lymph nodes from a colon cancer discovered during laparotomy.

Borderline tumours: leiomyoblastomas $(n=9)$ and leiomyomas $(n=4)$

SCTs of uncertain malignancy always corresponded to gastric tumours. The mean maximal diameter of the tumour was 49 (range 30-100) $\mathrm{mm}$. Five tumours were ulcerated $(38 \%)$.

All the leiomyoblastomas arose from the fourth echo poor layer, with frequent extragas-

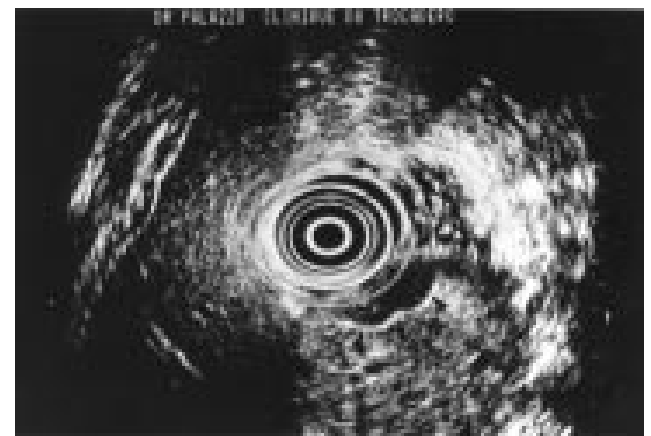

Figure 4 Gastric leiomyoblastoma (diameter $4 \mathrm{~cm}$ ) with cystic spaces (arrowheads).

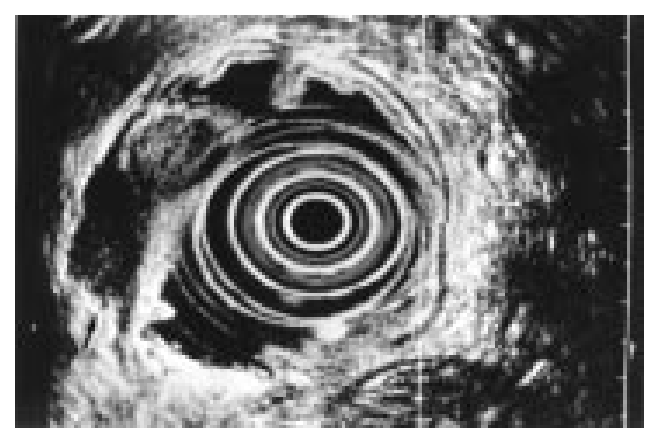

Figure 5 Gastric leiomyoblastoma (diameter $3 \mathrm{~cm}$ ) with irregular margins (arrow).

tric development (36\%). Their echostructure was often heterogeneous (67\%), with cystic spaces in five cases (56\%) (fig 4). The tumour margin was irregular in four cases (44\%) (fig 5). No suspect lymph nodes were observed. Cystic spaces were present or the margins were irregular in eight of nine leiomyoblastomas.

All four leiomyomas for which a benign nature could not be confirmed on the pathological specimen measured more than $30 \mathrm{~mm}$, while the extraluminal margins were irregular in two cases and a heterogeneous pattern without cystic spaces was found in two cases. In one case cystic spaces and a single satellite lymph node with a malignant pattern were observed. A large area of necrosis was found in the tumour on pathological examination. The lymph node corresponded to a large inflammatory node adjacent to the ulcerated necrotic tumour.

\section{STATISTICAL ANALYSIS}

Univariate analysis showed that extraluminal irregular margins, cystic spaces, a diameter greater than $30 \mathrm{~mm}$, exophytic development, and lymph nodes with a malignant pattern were associated with potential malignancy (table 1). Extraluminal irregular margins $(\mathrm{p}=$ $0.002)$ and cystic spaces in the tumour ( $\mathrm{p}=$ 0.001) were the two EUS features independently predictive of potential malignancy in logistic regression analysis $\left(R^{2}=0.6\right)$.

Table 2 shows the value of each EUS feature in predicting potential malignancy. The most predictive features were irregular margins, cystic spaces, and lymph nodes with a malignant pattern. Each criterion had a high positive predictive value but limited sensitivity. The presence of two of these three features in a 
Table 2 Sensitivity, specificity, and negative and positive predictive values of endoscopi ultrasonographic (EUS) features for potential malignancy - that is, leiomyosarcomas or stromal cell tumours of uncertain malignant potential

\begin{tabular}{lllll}
\hline & & & $\begin{array}{l}\text { Predictive } \\
\text { positive } \\
\text { value }\end{array}$ & $\begin{array}{l}\text { Predictive } \\
\text { negative } \\
\text { value }\end{array}$ \\
\hline Extraluminal irregular margins & 50 & 91 & 80 & 76 \\
Cystic spaces & 41 & 94 & 82 & 71 \\
Lymph nodes & 23 & 97 & 83 & 66 \\
Exophytic development & 36 & 88 & 67 & 68 \\
Maximal diameter $>30 \mathrm{~mm}$ & 77 & 59 & 56 & 83 \\
Ulceration & 54 & 70 & 54 & 70 \\
Heterogeneity (without cystic spaces) & 27 & 67 & 39 & 61 \\
\hline
\end{tabular}

Values are expressed as percentages.

Table 3 Frequency of at least one of the three most predictive features of potential malignancy (namely irregular margins, cystic spaces, and lymph nodes) in different types of stromal cell tumours

\begin{tabular}{llll}
\hline $\begin{array}{l}\text { Presence of at least one of the } \\
\text { predictive features in: }\end{array}$ & $\begin{array}{l}\text { Benign } \\
\text { leiomyomas }\end{array}$ & $\begin{array}{l}\text { Tumours of uncertain } \\
\text { malignant potential }\end{array}$ & Leiomyosarcomas \\
\hline All tumours & $4 / 34(12)$ & $12 / 13(92)$ & $8 / 9(89)$ \\
Tumours $\leqslant 30 \mathrm{~mm}$ in diameter & $2 / 20(10)$ & $3 / 3$ & $2 / 2$
\end{tabular}

Values in parentheses are percentages.

*Leiomyoblastomas and leiomyomas whose benignity could not be confirmed on the pathological specimen.

Table 4 Sensitivity, specificity, and negative and positive predictive values of endoscopic ultrasonographic (EUS) features for benignity of gastrointestinal stromal cell tumours

\begin{tabular}{lllll}
\hline & & & $\begin{array}{l}\text { Positive } \\
\text { predictive } \\
\text { value }\end{array}$ & $\begin{array}{l}\text { Negative } \\
\text { predictive } \\
\text { value }\end{array}$ \\
\hline Sens features & Sensitivity & Specificity & 61 & 69 \\
Diameter $\leqslant 30 \mathrm{~mm}$ & 89 & 69 & 80 & 40 \\
Homogeneous pattern & 53 & 70 & 78 & 41 \\
\hline
\end{tabular}

Values are expressed as percentages.

given tumour had a positive predictive value and specificity of $100 \%$ for potential malignancy, but only $23 \%$ sensitivity.

The presence of at least one of these three criteria had a sensitivity of $91 \%$, a specificity of $88 \%$, a positive predictive value of $83 \%$, and a negative predictive value of $94 \%$ for potential malignancy. Some $12 \%$ of benign leiomyomas presented one of these three features. At least one of these criteria was present in all malignant SCTs and in all SCTs of uncertain malignant potential measuring less than 30 $\mathrm{mm}$ in diameter (table 3 ).

In contrast, the three features most predictive of benignity were regular margins, diameter $\leqslant 30 \mathrm{~mm}$, and a homogeneous pattern (table 4). The combination of these three criteria had a sensitivity of $37 \%$ and a specificity and positive predictive value of $100 \%$ for benign SCTs.

\section{Discussion}

Gastrointestinal stromal cell tumours are often asymptomatic, usually being discovered incidentally during upper gastrointestinal endoscopy for unrelated conditions. Larger tumours may cause bleeding, abdominal pain, or obstruction. Before the advent of EUS, the management of SCTs was based on the estimated size of the tumour and the presence of manifestations attributable to the tumour. ${ }^{7} \mathrm{~A}$ presumptive diagnosis of SCT can now be made by using EUS. ${ }^{8-10}$ It is controversial whether gastrointestinal SCTs in asymptomatic patients should be removed to prevent complications or malignancy.
Histological assessment of malignancy in gastrointestinal SCTs is notoriously difficult, except in the case of invasion of adjacent structures or metastasis. ${ }^{12}$ The value of conventional histological signs such as dense cellularity and pleomorphism is controversial. The most useful variable is mitotic activity, but several grading systems are used. ${ }^{12}{ }^{11}$ The prognosis of gastric leiomyoblastomas is unclear. ${ }^{12}$ The reported rate of metastatic spread is between 3\% and 38\%. ${ }^{12}$ The main problem in the asymptomatic patient is to determine whether or not the SCT has malignant potential, and this is not always shown by histological analysis. In this study we individualised SCTs of uncertain malignant potential, which pathologists classify as a subgroup of tumours. ${ }^{12}$

Reviews of SCT studies by EUS reported until 1992 showed that certain features were suggestive of malignancy (larger nonhomogeneous tumours with irregular outer margins). However, EUS was not considered a reliable tool for differentiating between benign and malignant SCTs. ${ }^{49}$ More recently, two studies showed the usefulness of EUS in this indication. ${ }^{36}$ In contrast, the accuracy of endosonography guided fine needle aspiration biopsy is limited in gastrointestinal wall lesions. ${ }^{13}$ Yamada et $a l^{6}$ reported five EUS features predictive of malignancy: tumour diameter $\geqslant 30 \mathrm{~mm}$, nodular shape, depth of ulceration $(\geqslant 5 \mathrm{~mm}$ ), a heterogeneous pattern, and the presence of cystic spaces; however, the series included only four malignant tumours. Chak et $a l^{\beta}$ recently reported that four EUS features were helpful in predicting SCT malignancy, comprising size, irregular extraluminal borders, cystic spaces (greater than $4 \mathrm{~mm}$ ), and echogenic foci (greater than $3 \mathrm{~mm}$ ). Agreement on these features between observers was only fair to moderate, and was poor for irregular luminal borders.

We analysed the eight EUS features commonly recorded in our institution. Only irregular extraluminal margins and cystic spaces were independent predictors of potential malignancy. Tumour size is a reliable predictor of malignant potential. ${ }^{14}$ Moreover, in gastrointestinal leiomyosarcomas, larger lesions have a greater tendency than smaller ones to metastasise, ${ }^{14}$ but the large degree of size overlap rules out the use of this parameter alone to predict malignancy. ${ }^{12}$ In our series the mean size of benign leiomyomas was relatively large, as only histologically defined tumours were studied. This size was not representative of benign SCTs, as in our experience most asymptomatic patients with small tumours did not receive surgery. Most tumours project into the gastric lumen as polypoid submucosal growths and are prone to surface ulceration. Some SCTs protrude from the outer surface as exophytic subseral lesions, while a few may grow in both directions to produce a dumb bell appearance. Exophytic development and ulceration are more common in larger tumours. ${ }^{1}$ The low incidence of lymph node metastases of gastrointestinal leiomyosarcomas is well known. A review of published series gave nodal metastasis rates of $7-13 \% .{ }^{14}$ Our patient 
sample did not mirror this frequency, as four of the nine leiomyosarcomas (44\%) were associated with nodal metastases, but two of these patients had large nodal metastases which led to the discovery of the gastric leiomyosarcoma and probably accounted for this difference.

A combination of three criteria (regular extraluminal margins, diameter $\leqslant 30 \mathrm{~mm}$, and homogeneous pattern) had a sensitivity of $37 \%$ and a specificity and predictive positive value of $100 \%$ for benignity. In patients with such features, EUS may be a useful decision aid, particularly if they are at an increased risk of surgery or have lesions that are difficult to resect-for example, oesophageal tumours. However, this approach needs to be validated in a prospective study.

As in other such studies, our results should be interpreted in the light of technical limitations. ${ }^{3}$ Firstly, this was a retrospective study. Videotapes were not available for all the patients, as they were not recorded when lesions were small and had no EUS features of malignancy. Secondly, although EUS was performed in a single institution, patients came from several centres and were selected for surgery according to institutional criteria. Thirdly, the histological specimens were not all reviewed by the same pathologist. It should be noted, however, that the leiomyosarcomas and SCTs of uncertain malignancy had already been reviewed by referral pathologists from different university hospitals.

In conclusion, EUS is reliable in predicting the potential malignancy of SCTs. The three most predictive EUS features are irregular margins, cystic spaces, and lymph nodes with a malignant pattern. The presence of at least one of these criteria had a sensitivity of $91 \%$, a specificity of $88 \%$, a positive predictive value of $83 \%$, and a negative predictive value of $94 \%$ for potential malignancy. A combination of two of these three criteria had a positive predictive value and specificity of $100 \%$. In contrast, tumours of $30 \mathrm{~mm}$ or less, with regular extraluminal margins and a homogeneous pattern, are likely to be benign.

This work was presented in part at the annual meeting of the American Society for Gastrointestinal Endoscopy, May 1998, New Orleans, Louisiana, USA.

1 Campbell F, Bogomoletz WV, Williams GT. Tumours of the oesophagus and stomach. In: Fletcher CD, ed. Diagnostic histopathology of tumours. London: Churchill Livingstone, 1995:193-242.

2 Kempson RL, Hendrickson MR. Gastrointestinal stromal (smooth muscle) tumours. In: Whitehead R, ed. Gastrointestinal and oesophageal pathology. 2nd ed. Edinburgh: Churchill Livingstone, 1995:727-39.

3 Chak A, Canto M, Rösch T, et al. Endonographic differentiation of benign and malignant stromal cell tumours. Gastrointest Endosc 1997;45:468-73.

4 Rösch T, Classen M. Gastroenterological endosonography. Rosch T, Classen M.
Stuttgart: Thieme, 1992 .

5 Tio TL, Tytgat GNJ, Den Hartog Jaeger FCA. Endoscopic Tio TL, Tytgat GNJ, Den Hartog Jaeger FCA. Endoscopic
ultrasonography for the evaluation of smooth muscle ultrasonography for the evaluation of smooth muscle
tumours in the upper gastrointestinal tract: an experience tumours in the upper gastrointestinal tract: an exp
with 42 cases. Gastrointest Endosc 1990;36:342-50.

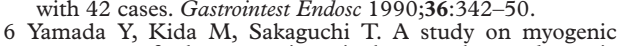
tumours of the gastrointestinal tract by endoscopic ultrasonography. Digestive Endoscopy 1992;4:396-408.

7 Palazzo L, Landi B, Cellier C, et al. Aspects échoendoscopiques des tumeurs bénignes de l'estomac. Encyclopédie Médico-Chirurgicale, Radiodiagnostic Appareil Digestif. Paris: Elsevier, 1996:33-120E10.

8 Caletti G, Zani L, Bolondi L, et al. Endoscopic ultrasonography in the diagnosis of gastric submucosal tumor. Gastrointest Endosc 1989;35:413-18.

9 Rösch T, Lorenz R, Dancygier H, et al. Endosonographic diagnosis of submucosal upper GI tract tumours. Scand $\mathcal{F}$ Gastroenterol 1992;27:1-8.

10 Yasuda K, Cho E, Nakajima M, et al. Diagnosis of submucosal lesions of the upper gastrointestinal tract by submucosal lesions of the upper gastrointestinal tract by 517-20.

11 Bedossa P, Martin E. Quoi de neuf sur les tumeurs conjonctives du tube digestif? Ann Pathol 1994;14:350-6.

12 Lee JS, Nascimento AG, Farnell MB, et al. Epithelioid gastric stromal tumours (leiomyoblastomas): a study of fifty-five cases. Surgery 1995;118:653-60.

13 Wiersema MJ, Vilmann P, Giovannini M, et al. Endosonography-guided fine-needle aspiration biopsy: diagnostic accuracy and complication assessment. Gastroenterology 1997;112:1087-95.

14 Coit D, Raff JH. Gastrointestinal leiomyosarcomas. In: Raaf JH, ed. Soft tissue sarcomas. St Louis: Mosby, 1993:139-50.

15 Lee MYT. Leiomyosarcoma of the gastrointestinal tract: Should we consider metastases to regional lymph nodes? $\mathcal{F}$ Surg Oncol 1980;15:319-21. 